\title{
Photonic Integrated Circuits for Communication Systems
}

\author{
Jozef CHOVAN ${ }^{1,3}$, František UHEREK ${ }^{1,2}$ \\ ${ }^{1}$ International Laser Centre, Ilkovičova 3, 84104 Bratislava, Slovakia \\ ${ }^{2}$ Faculty of Electrical Engineering and Information Technology, Slovak University of Technology, \\ Ilkovičova 3, 81219 Bratislava, Slovakia \\ ${ }^{3}$ OptoNet Slovakia, s.r.o., Detvianska Huta 215, 96205 Hriňová \\ jozef.chovan@ilc.sk, frantisek.uherek@stuba.sk
}

Submitted April 30, 2018 / Accepted April 30, 2018

\begin{abstract}
Photonic Integrated Circuits allow to meet the increasing demand of communication systems for internet which is growing at about $40 \%$ per year. This growth is driven mainly by increasing video traffic in the internet network. This growth is now further accelerated by mobile access, with video clients shipping on all smart phones and tablets, enabling video to be consumed more conveniently via network connections anywhere and anytime. This paper reviews several material platform of photonic integrated circuits and compares their performance. This paper also describes the new approaches in the design and fabrication of optical transceivers based on photonic integrated circuits for next terabit era.
\end{abstract}

\section{Keywords}

Integrated photonics, photonics integrated circuit, optoelectronics, optical transport network

\section{Introduction}

The development of optical transport network technology is stimulated by the emerging services such as data center cloud interconnection services, ultra-bandwidth video services, and $5 \mathrm{G}$ mobile network services will drive future optical communications industry development and architecture transformation.

A number of different industry surveys indicate that total internet demand is growing at about $40 \%$ per year. This growth is driven mainly by increasing video traffic in the network-Netflix now takes up to $30 \%$ of the internet's bandwidth at peak hours and new competitors like Amazon, Hulu, Youku, and the BBC iPlayer are growing rapidly. This growth is now further accelerated by mobile access, with video clients shipping on all smart phones and tablets, enabling video to be consumed more conveniently via network connections anywhere, anytime.

Monthly mobile data traffic per smartphone continues to increase in all regions. North America has the highest usage, and traffic is expected to reach 7.1 GigaBytes (GB) per month per smartphone by the end of 2017 and to in- crease to 48 GB by the end of 2023 . Western Europe has the second highest usage, with traffic set to reach 4.1 GB by the end of 2017 and 28 GB by the end of 2023 . Western Europe will be the region with the highest growth rate in monthly mobile data traffic per smartphone during the forecast period. The high average usage in India - estimated to reach $3.9 \mathrm{~GB}$ per month per smartphone at the end of 2017 - is mainly due to an introductory LTE offer by an operator during the latter half of 2016, which included free voice and data traffic. Data traffic is expected to continue to grow, reaching $18 \mathrm{~GB}$ per month per smartphone in 2023 [1].

Factors that will drive higher usage in general include an increase in the number of LTE subscriptions, improved device capabilities and more affordable data plans, as well as an increase in data-intensive content. As virtual reality and augmented reality technologies are more widely adopted, content will become even more data-intensive. Total mobile data traffic is expected to rise at a compound annual growth rate of 42 percent. Total mobile data traffic for all devices is anticipated to increase by 8 times during the forecast period, reaching around $110 \mathrm{~EB}$ per month by the end of 2023. At close to 85 percent, data traffic generated by smartphones is already accounting for the largest proportion of mobile data traffic. Going forward, smartphone data traffic will become even more dominant, and is expected to increase by 9 times during the forecast period to account for close to 95 percent of the total mobile data traffic by the end of 2023 [1].

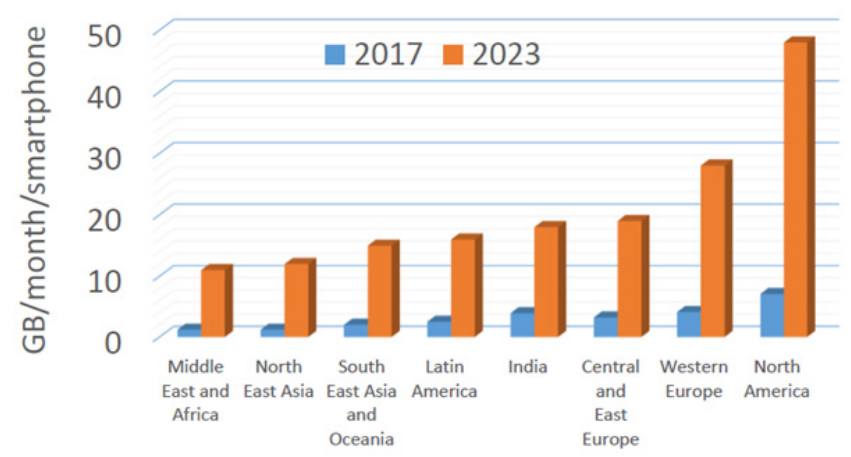

Fig. 1. Data traffic pre active smartphones in Gigabytes per month [1]. 


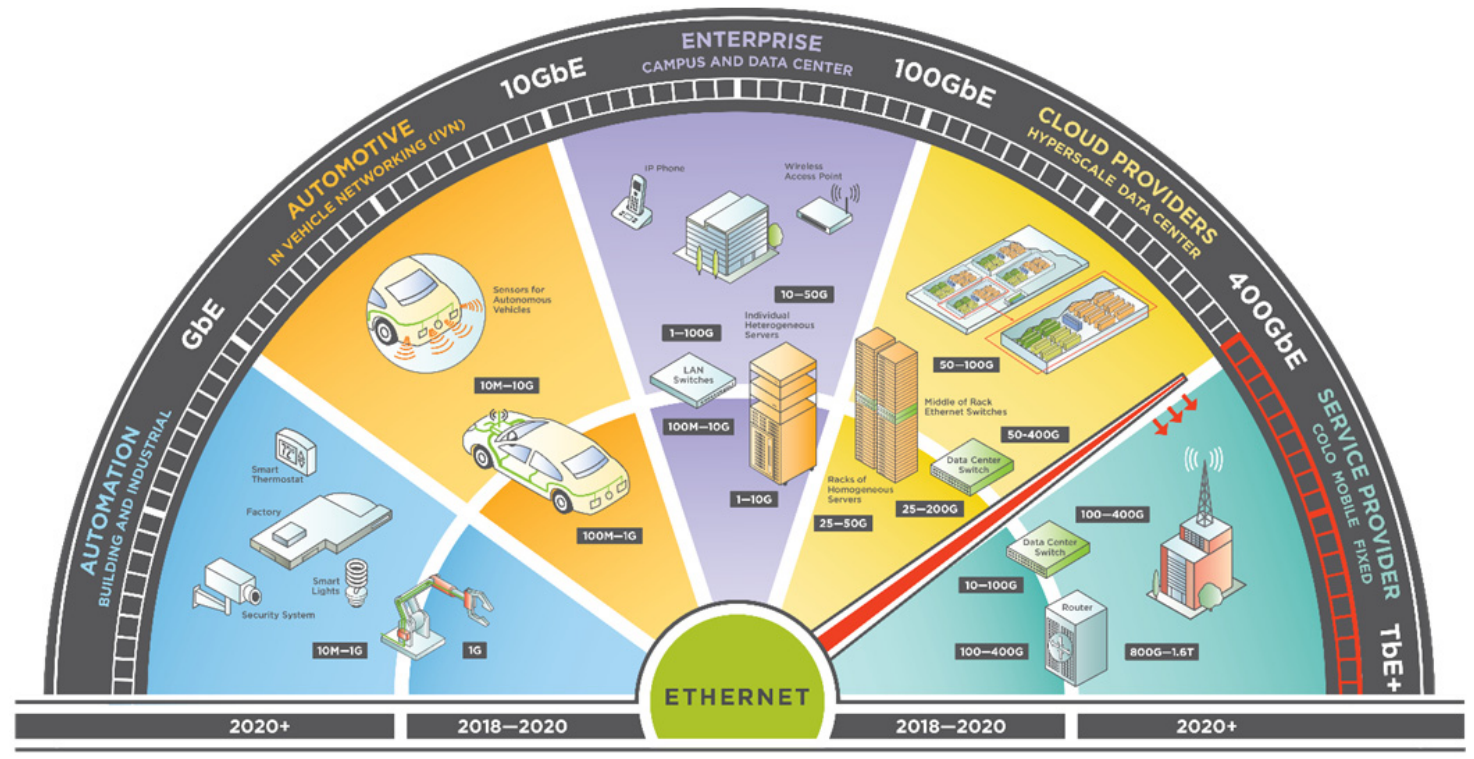

Fig. 2. The 2018 Ethernet Roadmap made by The Ethernet Alliance [2].

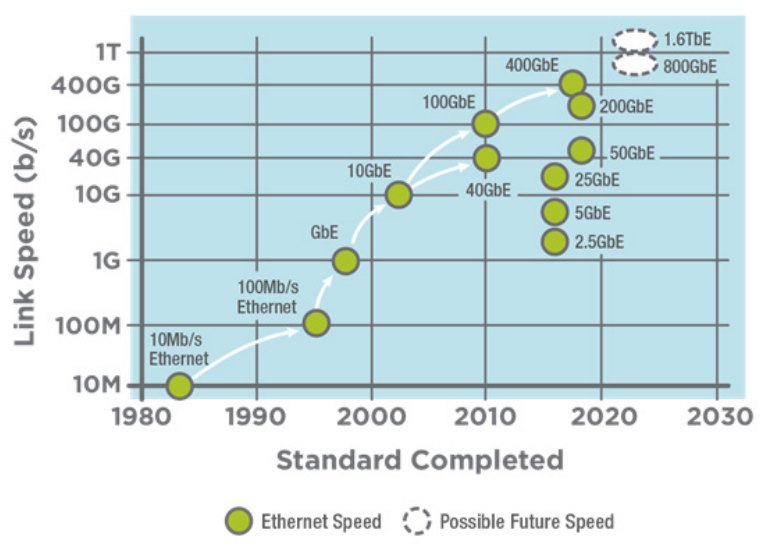

Fig. 3. The past, present and future of Ethernet speeds [2].

The Ethernet Alliance's new roadmap traces Ethernet's path from $10 \mathrm{Mb} / \mathrm{s}$ through present-day speeds of 1 to 400 gigabit Ethernet $(\mathrm{GbE})$, and looks ahead to future speeds achieving up to 1.6 terabits (TbE) and beyond (Fig. 2). Fig. 3 shows the evolution of the Ethernet speeds and possible future speeds [2]. The forward-looking map also provides guidance into key underlying technologies, current and future interfaces, and the numerous application spaces where Ethernet plays a fundamental role [2].

Building and industrial applications highlight the need for lower speed Ethernet solutions in harsh environments The Ethernet community is working to define a single standard for $10 \mathrm{Mb} / \mathrm{s}$ operation plus power delivery over a single twisted pair. This will consolidate a landscape of multiple legacy protocols, driving the promise of Ethernet's mufti-level interoperability to new heights for these spaces, as 2019 forecasts point to 165 million ports per year [2].

Automotive Ethernet is one of Ethernet's latest success stones. Forecasts predict up to 500 million ports of Ethernet will ship in 119 million vehicles by 2019. Ethernet links within cars provide data and power to reduce the cost and weight in vehicles while providing economies of scale and interoperability and the bandwidth demand of connected cards could be the next big driver for Ethernet to go beyond $400 \mathrm{GbE} \mathrm{[2].}$

Enterprise and campus applications drive the bulk of Ethernet port shipments with hundreds of millions of ports shipping per year. Ethernet's roots are in enterprise local area networks (LANs) where the entire Ethernet family, including the BASE-T products, can be found, LANs are rich in copper where over 70 Billion meters of cable have been deployed over the past 15 years. Enterprise data centers are very cost sensitive and most servers deploy GbE and $10 \mathrm{GbE}[2]$.

Cloud providers were the first to adopt $10 \mathrm{GbE}$ servers on a large scale in 2010 for hyperscale data centers. With voracious appetites for east-west traffic, hyperscale servers have moved to $25 \mathrm{GbE}$ today and will move to $50 \mathrm{GbE}$ by the end of 2018. Unique networking architectures within these warehouse scale data centers have driven multiple multimode and single-mode fiber solutions at 100 , 200 and $400 \mathrm{GbE}$. The bandwidth demands of hyperscale data centers and service providers continue to grow exponentially and in a similar direction that blurs the lines between the two [2].

Service providers have driven higher speed Ethernet solutions for decades. Router connections, client side optics for optical transport networks (OTN) equipment, and wireless backhaul have continually pushed Ethernet to higher rates and distances to meet the demands for wireless connectivity. And with global demand by consumers for video, this shows no signs of changing [2].

\section{Optical Communication Systems}

The optical fiber transmission data rate of a single wavelength channel has increased from $2.5 \mathrm{~Gb} / \mathrm{s}$ in 1985 to $400 \mathrm{~Gb} / \mathrm{s}$ in 2015 , representing a 160 -fold increase over 
30 years. The main technologies involved include high-speed electrical-optical modulation, high-speed optical detection, hard-decision forward error correction (HD-FEC), differential phase shift keying (DPSK) and differential quadrature phase-shift keying (DQPSK), coherent detection based on optical digital signal processing (ODSP), soft-decision forward error correction (SD-FEC), polarization-division multiplexing (PDM), quadrature amplitude modulation (QAM) and faster-than-Nyquist (FTN) modulation and demodulation. With the introduction of super-channel technology, the channel data rate can be further increased to beyond $1 \mathrm{~Tb} / \mathrm{s}$. At the transport system level, the introduction of broadband optical fiber amplifiers, such as erbium-doped fiber amplifiers (EDFA) and Raman amplifiers, makes wavelength-division multiplexing (WDM) a reality. The single-fiber transmission capacity of optical fiber communication has increased from $2.5 \mathrm{~Gb} / \mathrm{s}$ in 1985 to $20 \mathrm{~Tb} / \mathrm{s}$ in 2015 , representing a remarkable increase of 8000 times over the last 30 years. Additionally, optical fiber transmission links have also evolved from single span to today's multi-span links, transparent WDM network, and flexible-grid WDM. During the early development of optical communication systems, intensity modulation/direct detection (IMDD) was used to implement optical transmission, achieving transport speeds of up to $10 \mathrm{~Gb} / \mathrm{s}$. As the speed reached $40 \mathrm{~Gb} / \mathrm{s}$, the advantages of DPSK/DQPSK technology became apparent [3].

Today, in the $100 \mathrm{~Gb} / \mathrm{s}$ era, coherent optical technology is becoming the mainstream high-speed optical communication system. Currently, $100-\mathrm{Gb} / \mathrm{s}$ coherent-detection transceivers are in large-scale deployment and 400-Gb/s coherent-detection transceivers are commercially available. As the industry gradually advances toward $1 \mathrm{~Tb} / \mathrm{s}$ and beyond, new technologies such as super-channel transmission, large-scale photonic integration, high-level QAM, and FTN are being introduced. ODSP is a transport technology that marks the beginning of the digital era for the optical communication systems. ODSP is capable of compensating for chromatic dispersion, polarization rotation, polarization-mode dispersion, and certain fiber nonlinear impairments. It can also mitigate signal degradation due to bandwidth limitations of the transmitter and the receiver. Future ODSP promises to be even more powerful, especially with the availability of next-generation sub-10-nm or even sub5-nm CMOS technologies. Resource-intensive algorithms such as SD-FEC, fiber nonlinearity compensation, and others could be practically implemented. ODSP technology is expected to continue playing an important role in future high-speed long-haul optical transmission systems [3].

ODSP technology also enables more efficient utilization of high-capacity optical transmission systems through adaptive modulation with variable modulation bandwidth and adjustable modulation format. In flexible-grid WDM, channel spacing can be reduced from the typical $50 \mathrm{GHz}$ to $37.5 \mathrm{GHz}$ and $33.3 \mathrm{GHz}$, respectively increasing the number of C-band wavelength channels by 1.33 and 1.5 times to 128 and 144. For ODSP with 16-nm CMOS, which generally supports $100-\mathrm{Gb} / \mathrm{s}$ PDM-QPSK, $150-\mathrm{Gb} / \mathrm{s}$ PDM-8QAM, 200-Gb/s PDM-16QAM, and 400-Gb/s PDM-
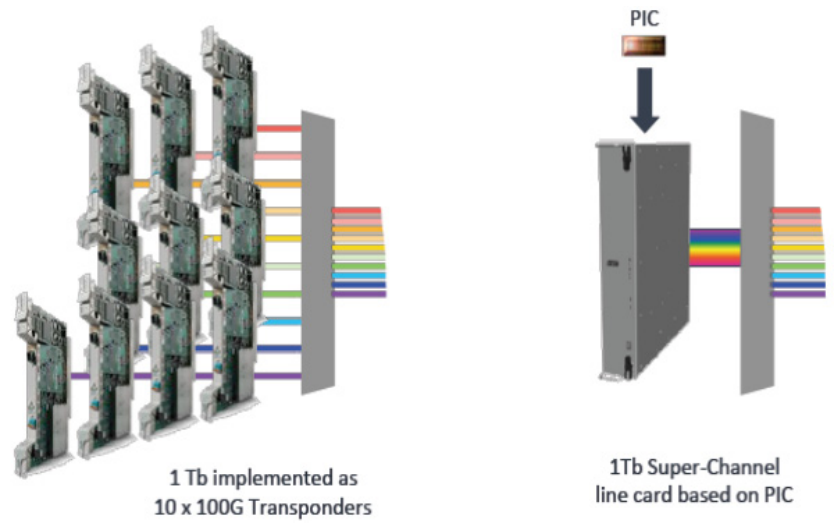

1Tb Super-Channel line card based on PIC

Fig. 4. A super-channel built with a PIC. The PIC enables hundreds of optical functions to be collapsed into two small chips the size of a fingernail enabling, in this example, $10 \times 100 \mathrm{~Gb} / \mathrm{s}$ in a single line card [4].

64QAM, the same hardware supports different transport distances and different modulation formats at different spectral efficiencies. When the transmitter and receiver both use ODSP, the transceiver can be software-defined, and the flexible-grid WDM can be supported [3].

Super-channels allow a Terabit of DWDM capacity to be turned up in a single operational cycle, without any penalty in terms of spectral efficiency and with the same optical reach as today's generation of $100 \mathrm{G}$ coherent transponders. It is clear that a 10 carrier super-channel requires 10 sets of optical components per line card. Implementing such an interface using discrete optical components would seem totally unrealistic. Figure 4 shows the scale of the problem. On the left we see 10 individual $100 \mathrm{G}$ transponders. These will contain in total around 600 optical functions that are probably implemented in discrete optical components. On the right of Fig .4 there is a Terabit super-channel line card. All of the major optical functions on all 10 100G line cards have been integrated into a single pair of PICs - one to transmit and one to receive. All 10 carriers can now be implemented brought into service in one operational cycle, consuming far less power than 10 discrete transponders and resulting in far greater service reliability. PICs bring the same kind of engineering practicality to super-channels that electronic integration brings to multicore CPUs or graphics engines. Within reason, the more carriers are in the super-channel, the simpler the electronics and the better the optical performance. PICs remove the limitation of optical component complexity, and allow the right engineering balance to be chosen [4].

\section{Photonic Integrated Circuit}

The integration of optical components and functions into a large scale PIC shows significant benefits when it is integrated into an optical communication system. It enables significant power, space and cost savings, new functionality and so new significant increasing transmission capacity of communication systems. PICs still are several orders of magnitude more expensive than their microelectronic counterparts, which has restricted their application to a few 
niche markets. In microelectronics there is a clear exponential development in the number of transistors per chip, which has been doubling every two years on average during the last four decades. This phenomenon is known as Moore's law. Figure 5 reveals a similar development in microphotonics, albeit in an early stage and with a much larger scatter than its microelectronic counterpart. If we restrict ourselves to devices based on AWGs, with a more or less comparable technology (AWGs with integrated amplifiers and/or detectors) most of the outliers disappear, however, which suggests that photonic integration is taking a similar development path to microelectronics, probably driven by the same improvements in process equipment [5].

Infinera company has pioneered the monolithic integration of hundreds of photonic functions, including lasers, modulators, waveguides and other optical components, into large-scale PICs, as well as the use of semiconductor manufacturing processes for PICs. PICs provide significant flexibility and efficiency benefits when integrated into DWDM systems. Meanwhile, electronic function integration has been progressing as CMOS (complementary metal-oxide semiconductor) technology continues to improve. As feature sizes have shrunk and design tools improved over the years, the maximum complexity possible in an application specific integrated circuit (ASIC) has grown from thousands of gates to several hundreds of millions. This level of integration delivers higher processing power in terms of GBaud (symbol rate) and bits per symbol, thereby allowing higher order modulation formats and coherent detection, ultimately resulting in better capacityreach performance. Infinera combines large-scale PICs with coherent ASICs, two important technologies for $500 \mathrm{~Gb} / \mathrm{s}$ and terabit super-channel transmission. In the first coherent era, Infinera's transmitter modulators were driven directly, and the optical impairments resulting from transmission were compensated for in the receiver by Infinera's FlexCoherent Processor. When this technology was introduced into the market, it enabled the move from $10 \mathrm{~Gb} / \mathrm{s}$ to $100 \mathrm{~Gb} / \mathrm{s}$ per channel, a tenfold increase in fiber capacity, and at the same time increased the typical optical reach from about 2,500 kilometers to about 4,500 kilometers.

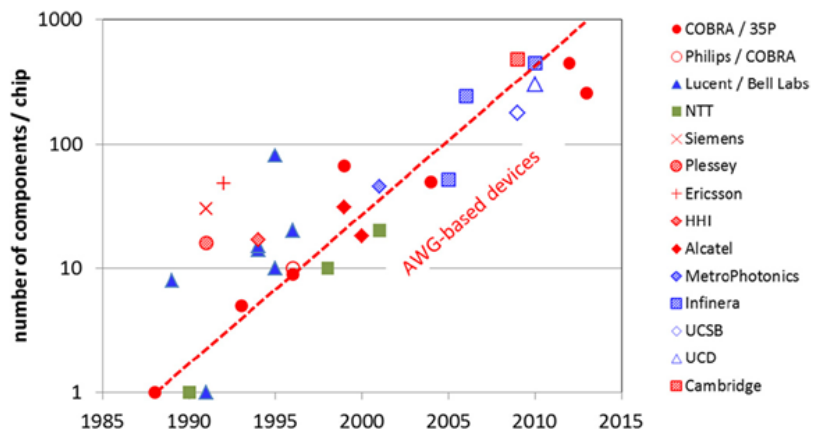

Fig. 5. Development of chip complexity measured as the number of components per chip [5].

Infinera's latest FlexCoherent design introduces transmitter based processing and enhances receiver-based capability. This is illustrated in Fig. 6, in which the transmitter modulators are now driven by the combination of an advanced DSP and a digital-to-analog converter (DAC), which improves spectral shaping. Blue color represents the signal processing in electrical domain and red color represents the signal processing in optical domain. Thanks to advances in ASIC technology, all transmitter and receiver functionality can be integrated into a single FlexCoherent Processor chip within the optical engine. This new design, combining electronic and photonic enhancements, introduces new capabilities, enabling operators to improve their fiber capacity reach performance [4].

Figure 7(a) shows a few components that we can make with passive waveguides. The most important ones are MMI couplers and AWG demultiplexers. With deepetched strong confinement waveguides we can make MMIreflectors and compact ring filters. Another important building block that we can make in a passive waveguide is a polarization converter. By placing it appropriately in a Mach-Zehnder interferometer (MZI) we can make polarization splitters and combiners, and by placing it halfway a polarization dependent component, the response of this component becomes polarization independent. SOAs in combination with passive devices offer a broad range of functionalities, as illustrated in Fig. 7(b): Fabry-Perot (FP) lasers, multi-wavelength lasers, ring lasers and, when used

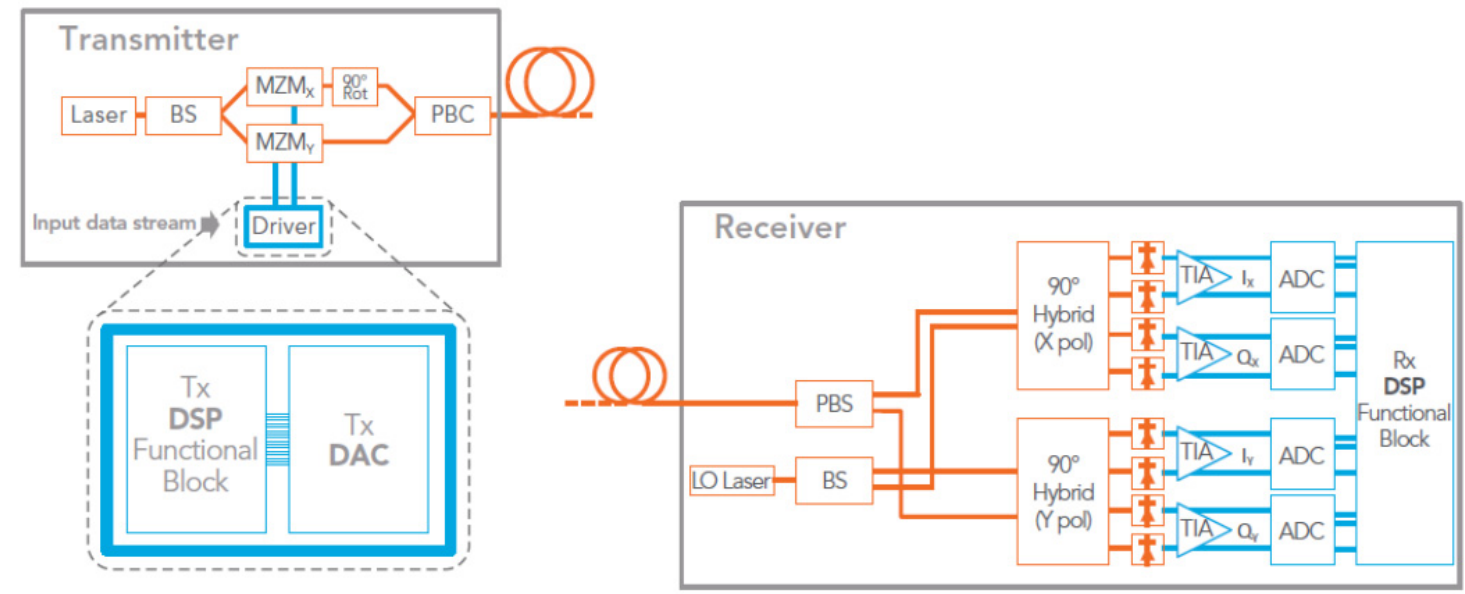

Fig. 6. Infinera FlexCoherent ${ }^{\mathrm{TM}} \mathrm{TX} / \mathrm{RX}$ Block Diagram [4]. 


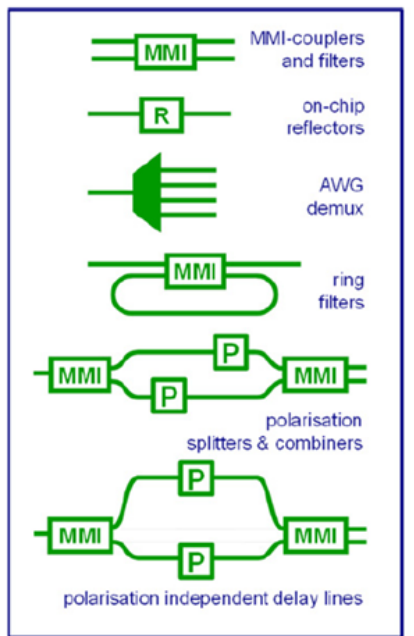

a) Passive Waveguide Devices

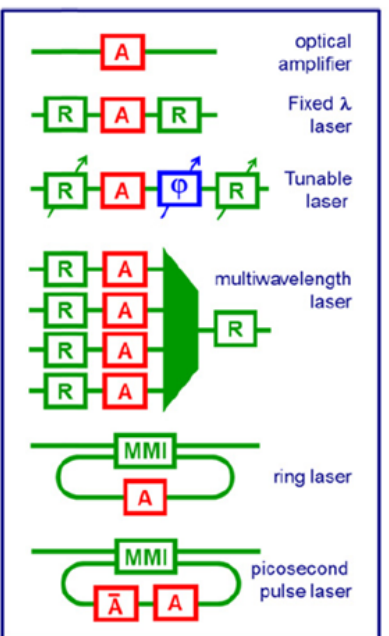

b) Lasers and amplifiers

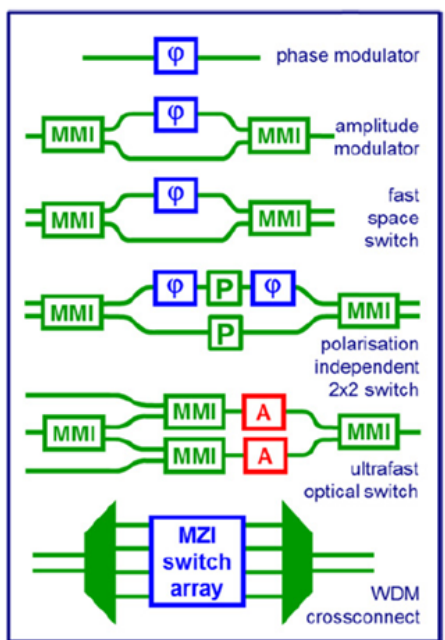

c) Switches and modulators

Fig. 7. Examples of the functionalities that can be realized with (a) passive waveguides devices alone, or in combination with (b) optical amplifiers and (c) phase modulators. [5].

(a)
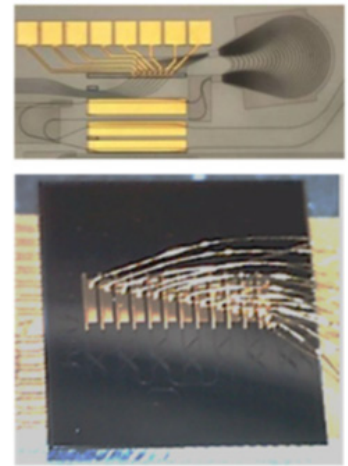

(b) (c)
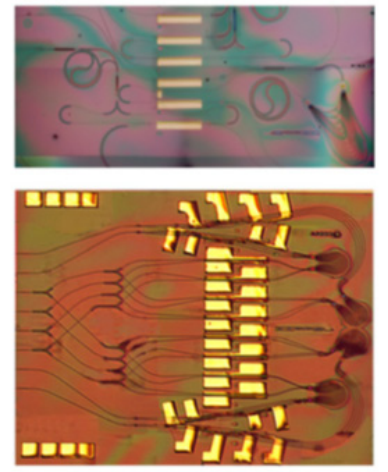

(d) (e)
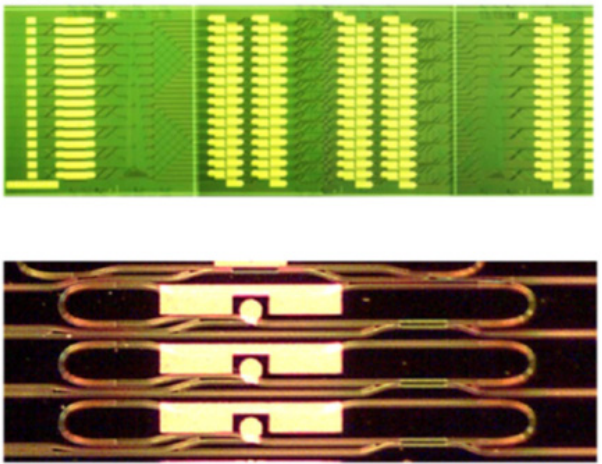

(f)

Fig. 8. Examples of PICs realized on the generic COBRA platform. (a) AWG-based discretely tuneable laser with nanosecond switching speed $\left(1.5 \times 3.5 \mathrm{~mm}^{2}\right)$; (b) $320 \mathrm{~Gb} / \mathrm{s}$ monolithic multistage SOA switching circuit, (c) multifunctional delay interferometer $\left(4 \times 2 \mathrm{~mm}^{2}\right)$; (d) $4 \times 4$ space and wavelength selective cross-connect $\left(4.2 \times 3.6 \mathrm{~mm}^{2}\right)$; (e) monolithic $16 \times 16$ photonic switch for broadband photonic packet-routing $\left(4.0 \times 13.2 \mathrm{~mm}^{2}\right)$; (f) broad frequency comb laser $\left(0.3 \times 2.2 \mathrm{~mm}^{2}\right)$ [5].

in combination with a wavelength tuneable reflector, also tuneable lasers. By using a short SOA section in reverse bias as a securable absorber we can make picosecond pulse lasers. And a SOA in reverse bias can also be used as a detector. Figure 7(c) illustrates some of the functionalities that we can make by combining phase modulators with passive devices: amplitude modulators, space switches, wavelength selective switches, such as WDM cross-connects and add-drop multiplexers. And by making use of the nonlinear properties of SOAs integrated in an MZI we can make ultrafast switches. Figure 8 shows and describes examples of PICs realized on the generic COBRA platform [5].

\subsection{Material Platform Technologies of PIC}

Table 1 displays the three major material platform technologies of PIC. InP offers state of the art performance for optoelectronic devices operating in the 1300-1600 nm wavelength window. As the information rates required from transceivers continue to increase, more sophisticated monolithic integration techniques are being deployed both within the discrete devices themselves and also in the creation of advanced integrated transmitter and receiver circuits. Band-gap engineering through epitaxial regrowth and selective area growth allow unprecedented chip-level function and performance in an ever-decreasing footprint. Lasers are combined with modulators, amplifiers, multiplexers, detectors and hybrids in wafer-scale processes [6].

Silicon photonics $(\mathrm{SiP})$ research can be dated back to the 1980s. However, the previous decade has witnessed an explosive growth in the field. $\mathrm{SiP}$ is a disruptive technology that is poised to revolutionize a number of application areas, for example, data centers, high-performance computing (HPC). The key driving force behind $\mathrm{SiP}$ is the ability to use CMOS like fabrication resulting in high-volume production at low cost and possible monolithic integration with CMOS electronics. This is a key enabling factor for 


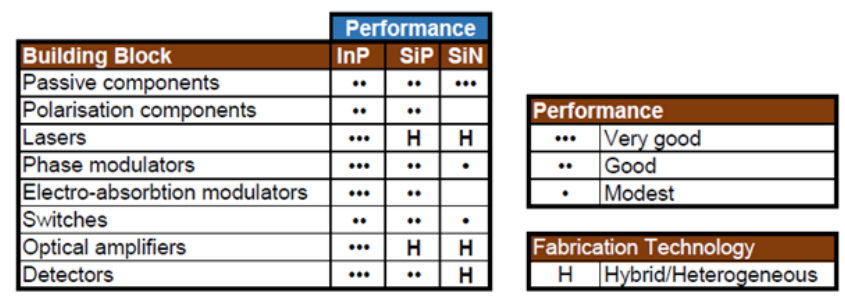

Tab. 1. Performance comparison between three major material platform technologies of integrated photonics [6].

bringing photonics to a range of technology areas where the costs of implementation using traditional photonic elements such as those used for the telecommunications industry would be prohibitive. SiP is entering a phase of increased commercialization and manufacturing growth in datacenter and HPC communication markets, due to the cost and power efficiency that high-density integration and WDM bring [7]. The SiP working spectral range is from $1.1 \mu \mathrm{m}$ up to $2 \mu \mathrm{m}$. This spectral range is possible extended by Ge on $\mathrm{Si}$ up to $8 \mu \mathrm{m}$ without leaving the CMOS technology.

SiN material platform is a versatile dielectric waveguide platform, called TriPleX and patented by LioniX International, which is based on alternating silicon nitride and silicon dioxide films. Fabrication with CMOS-compatible equipment based on low-pressure chemical vapor deposition enables the realization of stable material compositions being a prerequisite to the control of waveguide properties and modal shape. The transparency window of both materials allows for the realization of low-loss waveguides over a wide wavelength range $(400 \mathrm{~nm}-2.35 \mu \mathrm{m})$. Propagation losses as low as $5 \times 10^{-4} \mathrm{~dB} / \mathrm{cm}$ are achieved [8].

PIC enabled products outperform equivalent combinations of discrete components at the functional level. From Tab. 1 it is seen that InP and SiN are perfectly complementary. A successful hybrid platform integrating InP and SiN components will offer, therefore, superior performance for both active and passive components and is a very promising approach for complex PICs which require very low propagation losses, e.g. in delay lines or high-Q filters. Ultra-low linewidth lasers by combining InP and TriPleX chips from LioniX and Fraunhofer HHI have recently been reported [9]. The TriPleX platform will not only allow the combination of efficient InP lasers with ultra-low loss passive waveguides, but it will also enable testing of InP circuits on wafer scale by using TriPleXbased optical waveguide probes.

\subsection{Future Technology Nodes}

As PIC technology development continues, novel schemes will emerge either to enable new applications by combining different technologies in a reliable way or to continue scaling the density of components for VLSI photonic systems. Research lines are targeting new technology nodes within a period of five to ten years.

For both $\mathrm{SiP}$ and $\mathrm{SiN}$, lasers and amplifiers have to be integrated in a hybrid way. By hybrid integration we under- stand an approach in which different chips are coupled after processing. Hybrid integration technology has made considerable progress. It is especially favorable where the PIC requires component properties which cannot be provided by a single platform, for example optical gain and very low loss. The integration of InP active components (lasers and modulators) is on the roadmap of LioniX International, and will be implemented in the TriPleX PDK and offered in MPW services [6].

By heterogeneous integration we understand an approach in which an unprocessed or partially processed wafer or die of different materials, e.g. InP is bonded to a processed wafer, e.g. silicon photonics or CMOS, and further processed on wafer scale after bonding. Heterogeneous integration of InP lasers and amplifiers on SiP circuits offers more flexibility in placement of lasers and amplifiers than hybrid integration, at the cost of added complexity in the fabrication process, because processing of both InP and SiP circuitry is now required. Further, the coupling between the InP and the SiP layer introduces coupling losses of $1 \mathrm{~dB}$ or more, and the coupling structures require significant space, of in the order of $100 \mu \mathrm{m}$ per coupler depending on the substrate design. Heterogeneous integration of InP-Photonics on Silicon Electronics avoids optical coupling losses as only one PIC technology is used, and offers high-performance electrical connections. The approach consists in adapting the generic InP photonic integration process such as to make it suitable for wafer scale bonding onto a (Bi) CMOS wafer in which the driver, receiver and control electronics are integrated. The bonding is achieved by means of a polymer layer that is optically and thermally insulating, and thermal, mechanical and electrical connections are made with vias through the bonding layer [6].

The InP membrane on Silicon (IMOS) platform aims at further reducing the footprint of photonic devices by moving towards a high contrast waveguide technology embedded in an InP membrane that can be fabricated on silicon wafers. It has seen significant progress since the start of the technology and now offers a range of passive waveguide components such as MMIs, ring resonators, directional couplers, dielectric and metallic grating couplers, and polarization converters. [6].

Silicon photonics technology is leading an epochmaking technology transformation of the optical network industry from the discrete-component era into an integrated-chip era that features automation and large-scale production. The influence of the technology is akin to the impact on the electronic circuit industry caused by the transformation from the electron tube era to the transistor integrated circuit era.

Graphene, a type of two-dimensional material, is currently used on photonic components in research phase. Compared with silicon, graphene can theoretically bring higher bandwidth, low drive voltage, and smaller dimensions. Additionally, the manufacturing of graphene components is compatible with the silicon-based CMOS process. Therefore, the current silicon photonics technology and 
process can be used for manufacturing graphene components. Using graphene materials in photonic components means combining the advantages of the two types of materials, implementing next-generation beyond-silicon photonics technology. In the future, silicon photonics technology working with graphene materials can achieve various optical switching, optical routing, optical logic, optical storage, and optical signal processing functions of nextgeneration all-optical networks. These components are superior in implementing a new optical manipulation mechanism and have the potential to change the component types and structures of existing optical networks, dramatically simplifying optical networks and implementing revolution of the optical communications industry. Optical packet switching networks with optical memories will be possible realized.

\section{Conclusion}

The paper reviews the main application for increasing the demand on internet traffic and for coming the terabit era in optical transport network, in cloud and in high performance computing systems. The new technologies and signal processing in coherent optical transport network based on photonic integrated circuit are described. The properties and performance of the three main material platform of photonic integrated circuit and their future technology nodes are reviewed.

\section{Acknowledgments}

The work was supported by projects 1/0907/13 of VEGA grant agency of the Ministry of Education, Science, Research and Sport of the Slovak Republic, projects APVV-14-0716 and SK-AT-2017-0005 from the Slovak Research and Development Agency of the Ministry of Education, Science, Research and Sport of the Slovak Republic.

\section{References}

[1] ERICSSON Mobility Report November 2017, Mobile data traffic growth outlook. [Online] Available at: https://www.ericsson.com/en/ mobility-report/reports/november2017/mobile-data-traffic-growth-outlook

[2] ETHERNET ALLIANCE. The 2018 Ethernet Roadmap. [Online] Available at: https://ethernetalliance.org /the-2018-ethernetroadmap/
[3] HUAWEI TECHNOLOGIES CO., LTD. White Paper on Technological Developments of Optical Networks. [Online] Available at: http://www.huawei.com

[4] INFINERA CORPORATION. The Next Generation of Coherent Optical. [Online] Available at: https://www.infinera.com/auth/?pid=7366

[5] SMIT, M., LEIJTENS, X., AMBROSIUS, H. et al. An introduction to InP-based generic integration technology. Semiconductor Science and Technology, 2014, vol. 29, no. 8, p. 1-41. DOI: 10.1088/0268-1242/29/8/083001

[6] THE JEPPIX CONSORTIUM. JePPIX Roadmap 2018. [Online] Cited March 2018. Available at: http://www.jeppix.eu/vision/

[7] THOMSON, D., ZILKIE, A., BOWERS, J. E., et al. Roadmap on silicon photonics. Journal of Optics, 2016, vol. 18, no. 7, p. 1-20. DOI: $10.1088 / 2040-8978 / 18 / 7 / 073003$

[8] WÖRHOFF, K., HEIDEMAN, R. G., LEINSE, A., et al. TriPleX: a versatile dielectric photonic platform. Advanced Optical Technologies, 2015 , vol. 4, no. 2, p. 189-207. DOI: 10.1515/aot2015-0016

[9] WALLACE, J. Integrated-Photonics Laser Has Record-Narrow Chip-Based Linewidth of $290 \mathrm{~Hz}$. [Online] Available at: http://www.laserfocusworld.com /articles/2017/07/integratedphotonics-laser-has-record-narrow-chip-based-linewidth-of-290hz.html

\section{About the Authors ...}

Jozef CHOVAN (corresponding author) was born in 1974. He graduated from the Slovak University of Technology in Bratislava with a MSc. and $\mathrm{PhD}$. degree in Optoelectronics and Optical Communication. His Ph.D. work focused on optical CDMA was awarded by Werner von Siemens Excellence Award 2003. Since 2004, working with the ILC in the design and characterization of photonic components and structures for photonic integrated circuits. Within the EU project PARADIGM submitted the design photonic integrated circuit for MUX/DEMUX optical CDMA system.

František UHEREK was born in 1954. He obtained his master and Ph.D. degree from the Slovak University of Technology in Bratislava. He was personally involved in establishing of the International Laser Centre (ILC) in Bratislava; he is actually the director of ILC. He is a wellknown expert in field of photonics, lasers and optical communication systems. He published more than 150 scientific papers in journals and in proceedings from scientific conferences. He is actively working on dissemination of current knowledge in field of electronics, photonics and lasers in Slovakia. 